\title{
Therapeutics
}

\section{A geriatric evaluation and management programme prevented functional decline and reduced depression in high risk older adults}

Boult C, Boult LB, Morishita L, et al. A randomized clinical trial of outpatient geriatric evaluation and management.J Am Geriatr Soc 2001 Apr;49:351-9.

QUESTION: Can an outpatient geriatric evaluation and management (GEM) programme prevent functional decline and reduce depressive symptoms and Medicare costs in high risk older adults?

\section{Design}

Randomised \{allocation concealed $*\}$, blinded (outcome assessors)*, controlled trial with 18 months of follow up.

\section{Setting}

Ramsey County and adjacent zip codes, Minnesota, USA.

\section{Patients}

568 patients who were $\geqslant 70$ years of age (mean age $79 \mathrm{y}$, $56 \%$ men), community dwelling, and Medicare beneficiaries; and were at high risk for hospital admission and functional decline. Exclusion criteria included living in a nursing home, illness requiring frequent physician visits, and communication barriers. Follow up was $97 \%$.

\section{Intervention}

Patients were allocated to GEM ( $\mathrm{n}=294)$, consisting of a home visit by a social worker, 2 visits to the GEM clinic, and 6 months of management by an interdisciplinary team, or usual care $(n=274)$ for a mean 6 months. A team comprising a geriatrics nurse practitioner, a geriatrician, a social worker, and a nurse assessed patients, set intervention priorities, created a plan of care, and provided comprehensive care. Patients had monthly clinic visits and regular telephone calls between visits.

\section{Main outcome measures}

Functional ability (Sickness Impact Profile: Physical Functioning Dimension [SIP:PFD], bed disability days [BDDs], and restricted activity days [RADs]); depressive symptoms (Geriatric Depression Scale [GDS]); and use and cost of healthcare services (Medicare payments)

\section{Main results}

Analysis was by intention to treat. After 18 months of follow up, the GEM group had fewer BDDs $(\mathrm{p}<0.05)$ than the control group but RAD values were similar $\{\mathrm{p}=0.71\} \ddagger$ (table). After 18 months of follow up, more patients in the usual care group than the GEM group experienced functional decline ( $\geqslant 3$ point increase in SIP:PFD score) and had possible depression (GDS score $\geqslant 11$ ) (table). After adjusting for baseline use of services, home care use at 12 months was less for GEM than for usual care (adjusted OR 0.60, 95\% CI 0.37 to 0.98 ). Groups did not differ for nursing home use or total Medicare costs (US\$11 $354 v$ US $\$ 11786, \mathrm{p}=0.93$ ).

\section{Conclusion}

A geriatric evaluation and management programme prevented functional decline and reduced bed disability days, depressive symptoms, and home care use, but did not reduce Medicare costs.
*See glossary.

†p Value calculated using data in article.
†Information provided by author. try 2000;34:748-54. recommendations. J Am Geriatr Soc 1999;47:269-76.
Sources of funding: National Institute on Aging and the Agency for Health Care Policy and Research; Health Care Financing Administration, American Academy of Family Physicians Foundation.

For correspondence: Dr C Boult, Lipitz

Center for Integrated Health Care,Johns Hopkins University, 624 North Broadway Street \#653, Baltimore, $M D$ 21205, USA. Fax +1 4109550470

A modified version of this abstract appears in Evidence-Based Nursing.
Geriatric evaluation and management (GEM) v usual care (UC) in high risk older adults§

\begin{tabular}{|c|c|c|c|c|}
\hline Outcomes at 18 months & GEM & UC & RRR $(95 \% \mathrm{CI})$ & NNT (Cl) \\
\hline Loss of functional ability & $40 \%$ & $52 \%$ & $24 \%(8.8$ to 37$)$ & $9(5$ to 24$)$ \\
\hline Depression & $8.8 \%$ & $18 \%$ & $52 \%(25$ to 69$)$ & 11 (7 to 27$)$ \\
\hline & \multicolumn{2}{|c|}{ Mean scores } & \multicolumn{2}{|c|}{ Mean difference $(\mathbf{C l})$} \\
\hline Bed disability days & 0.6 & 1.5 & 0.90 (0.22 to 1.6 & \\
\hline Restricted activity days & 2.1 & 2.3 & $0.20(-0.86$ to 1 . & \\
\hline
\end{tabular}

$\S$ Abbreviations defined in glossary; RRR, NNT, mean difference, and $\mathrm{Cl}$ calculated from data in article INot statistically significant.

\section{COMMENTARY}

Mental health services for older people have developed in response to the demographic imperative of an ageing population, but without an evidence base; however, there is some support for the notion that specialised psychogeriatric services deliver better quality care. ${ }^{1}$

This study by Boult $e t$ al provides data in support of carefully targeted outpatient assessment and specific management in geriatric medicine, which may be useful in old age mental health.

The traditional concept of comprehensive geriatric assessment (CGA) ${ }^{2}$ and recen evidence that treatment adherence after CGA could be enhanced ${ }^{3}$ are developed further by the authors' suggestion that GEM was likely to be most effective if the process was carried out by the same team.

The use of a small, expert, multidisciplinary team in the assessment and management of high risk patients over the short to medium term was effective in reducing functional decline, as well as disability bed days and home care use. The reason for the reduced rate of depression in those in the GEM group was not clear, but may have been related to the benefits of reduced functional decline, or to the non-specific effects of participation in the experimental group.

Two potential confounding variables may have lessened the differences in outcomes between the 2 groups; specifically, the control group's high risk status was disclosed to their primary care physicians, and they lived in an area known for progressive medical care.

Despite this, the study clearly showed the benefits of targeted assessment and focused intervention over a limited period by a small, expert team. It offers a useful service delivery model for clinicians confronted by large numbers of elderly patients and limited resources.

David Burke, BMed, MRCPsych, FRANZCP New South Wales Institute of Psychiatry, Sydney, New South Wales, Australia

1 Hickie I, Burke D, Tobin M, et al. The impact of the organisation of mental health services on the quality of assessment provided to older patients with depression. Aust NZJ Psychia-

2 Williamson J, Stokoe IH, Gray S, et al. Old people at home: their unreported needs. Lancet 1964;1:1117-20.

3 Reuben DB, Frank JC, Hirsch SH, et al. A randomized clinical trial of outpatient comprehensive geriatric assessment coupled with an intervention to increase adherence to 